\title{
Low Temperature Hot-Wire Polysilicon Waveguides
}

\author{
Taha M. Ben Masaud*, Antulio Tarazona ${ }^{* * *}$, Xia Chen ${ }^{* *}$, Graham Reed**, and H. M. H Chong* \\ *Nano Research Group, Electronics and Computer Science, University of Southampton, SO17 1BJ UK \\ ** Optoelectronics Research Centre, University of Southampton, SO17 1BJ UK \\ **** Echerkon Technologies \\ e-mail: tbm206@ecs.soton.ac.uk.
}

\begin{abstract}
We fabricated and measured low loss polysilicon waveguides deposited using Hot-Wire Chemical Vapor Deposition (HWCVD) at $240{ }^{\circ} \mathrm{C}$. The optical propagation loss was measured to be $11.9 \mathrm{~dB} / \mathrm{cm}$ at $\lambda=1550 \mathrm{~nm}$.
\end{abstract}

\section{INTRODUCTION}

Silicon photonics technology can offer an alternative to wired interconnections on LSI circuits by using ultra-compact silicon waveguides. The high bandwidth and small footprint of silicon waveguides have the potential to improve the performance of LSI circuits [1]. Silicon on insulator (SOI) has been the dominant development platform for silicon photonics technology due to its low optical propagation loss. However, it is constrained by the need for integration of multi-layered silicon photonics to the more established CMOS technology. Recently, there has been an increasing interest in polysilicon waveguides due to their low cost and added design flexibility [2]. However, most of the reported polysilicon films were deposited or post-treated at high temperatures $\left(\geq 900{ }^{\circ} \mathrm{C}\right)$ which may not be compatible with the CMOS fabrication process. Therefore, we propose polysilicon waveguides with low loss and low thermal budget achieved using hot-wire chemical vapour deposition (HWCVD).

An important characteristic of polysilicon waveguides is their low-cost compared to SOI. However, the high optical propagation losses $(\sim 1700 \mathrm{~dB} / \mathrm{cm})$ that were reported in early studies abated the interest of using polysilicon in photonics applications [3]. Engineering the waveguide structure and deposition processes has resulted in reducing the optical propagation loss to $\sim 6.45 \mathrm{~dB} / \mathrm{cm}$ for TE mode [4]. However, annealing polysilicon at temperatures $\geq 900{ }^{\circ} \mathrm{C}$ has been widely used to improve the crystallinity of the as-deposited films. Takei et al. reported low-loss silicon waveguides using hydrogenated microcrystalline silicon $(\mu \mathrm{c}-\mathrm{Si}: \mathrm{H})$ deposited at $250{ }^{\circ} \mathrm{C}$ [5]. Although a loss as low as $6.5 \mathrm{~dB} / \mathrm{cm}$ was reported for TE mode, hydrogenated microcrystalline silicon $(\mu \mathrm{c}$ $\mathrm{Si}: \mathrm{H})$ should still have higher optical loss than polysilicon due to its smaller grain size and amorphous nature. To avoid high deposition temperature, we propose Hot-Wire Chemical Vapor Deposition (HWCVD) instead of the more conventional Plasma-Enhanced Chemical Vapor Deposition (PECVD) and Low-Pressure Chemical Vapor Deposition LPCVD processes. Polysilicon HWCVD can be deposited at temperatures as low as $300{ }^{\circ} \mathrm{C}$ and can result in reduced ion-induced damage, high deposition rate, improved uniformity, and low-cost polysilicon films [7].

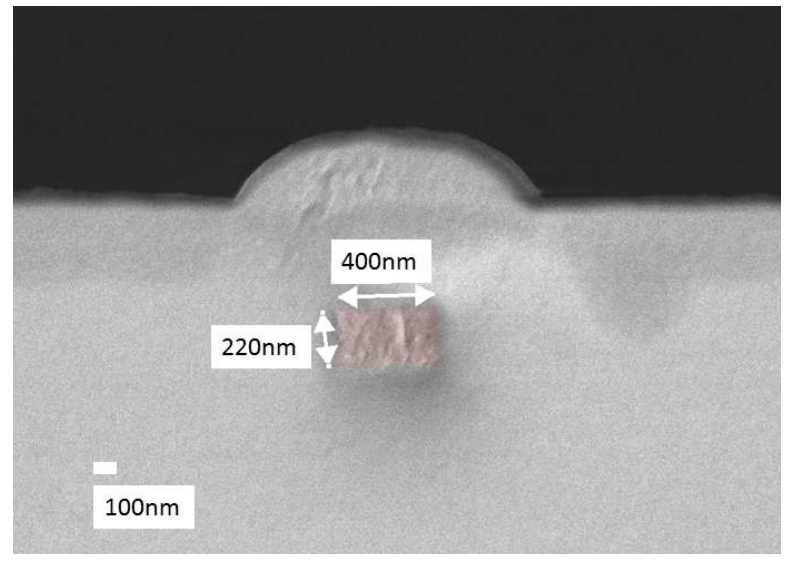

Fig. 1. An SEM cross-section of the polysilicon waveguide covered with PECVD $\mathrm{SiO}_{2}$

\section{EXPERIMENT AND RESULTS}

Thin polysilicon films (230 nm thick) were deposited using a Hot-Wire Chemical Vapor Deposition (HWCVD) system (Echerkon Nitor 301) at a temperature of $240{ }^{\circ} \mathrm{C}$ on top of a $2000 \mathrm{~nm}$ thick PECVD silicon dioxide $\left(\mathrm{SiO}_{2}\right)$ on a silicon wafer. Sub-micron waveguides were then patterned using e-beam lithography and conventional RIE etch. The waveguides were then covered with $700 \mathrm{~nm}$ PECVD $\mathrm{SiO}_{2}$ that was deposited at a temperature of $350{ }^{\circ} \mathrm{C}$. The surface roughness of the deposited polysilicon film was characterized using atomic force microscopy (AFM). The RMS value of the surface roughness was $8 \mathrm{~nm}$. Figure 1 shows an SEM image of a polysilicon waveguide $(400 \mathrm{~nm})$ covered with PECVD $\mathrm{SiO}_{2}$. The roughness of the cross-section and the top surface is a consequence of poly-crystalline grain. Raman scattering measurement with a $532 \mathrm{~nm}$ excitation wavelength was used to estimate the crystalline volume of the HWCVD polysilicon. The crystalline volume fraction, $X_{c}$, of the film was calculated using [6]

$$
X_{c}=\frac{I_{c-S i}}{I_{c-S i}+I_{a-S i}}
$$

where $I_{c-S i}$ and $I_{c-S i}$ are the sum of deconvoluted intensities for the c-Si peak centred at $520 \mathrm{~cm}^{-1}$ and the a-Si peak centred at $480 \mathrm{~cm}^{-1}$, respectively. The crystalline volume was found to be $>91 \%$. Moreover, the transmission properties of the fabricated HWCVD polysilicon waveguides were 


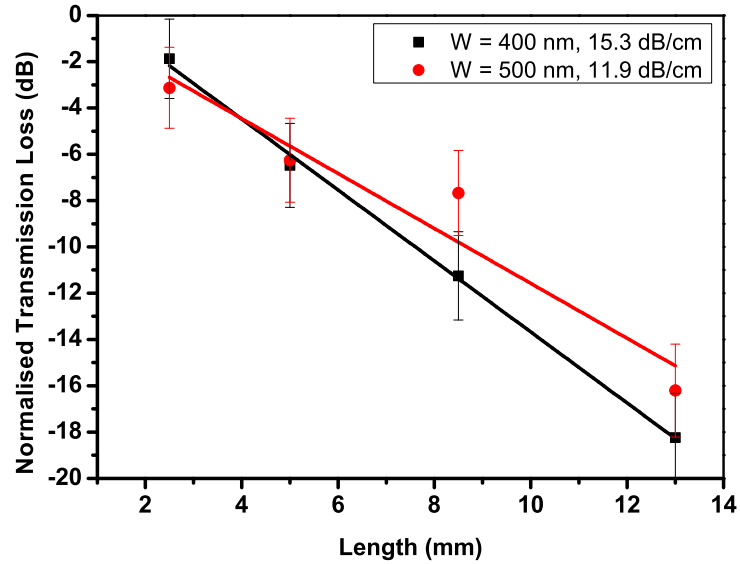

Fig. 2. (b) Transmission characteristics of the TE mode of the polysilicon waveguides of widths $400 \mathrm{~nm}$ and $500 \mathrm{~nm}$.

characterised at a wavelength of $1550 \mathrm{~nm}$. The polysilicon waveguides had lengths of $1,2.5,5,10$ and $13 \mathrm{~mm}$ and widths of 400 and $500 \mathrm{~nm}$. The propagation loss of the TE mode of the $400 \mathrm{~nm}$ was $15.3 \pm 0.3 \mathrm{~dB} / \mathrm{cm}$, while that of the $500 \mathrm{~nm}$ wide waveguide was $11.9 \pm 0.3 \mathrm{~dB} / \mathrm{cm}$. Figure 2 shows the transmission loss of the $400 \mathrm{~nm}$ and the $500 \mathrm{~nm}$ waveguides where the loss in $\mathrm{dB}$ was normalised to a length of $1 \mathrm{~mm}$. The relatively high surface roughness is expected to contribute most to the total propagation loss. Smoothing the surface using chemical mechanical polishing (CMP) should result in decreasing the loss to single figures.

\section{CONCLUSION}

In summary, we report on low loss $(11.9 \pm 0.3 \mathrm{~dB} / \mathrm{cm})$ polysilicon waveguides deposited using HWCVD at $240{ }^{\circ} \mathrm{C}$. Further study is being conducted to quantitatively estimate the contribution of the material-induced and scattering losses. Surface smoothing by means of CMP is being investigated to improve the optical propagation loss.

\section{ACKNOWLEDGMENT}

The author would like to thank the Libyan Ministry of Higher Education and the University of Southampton for sponsoring this project. The author would also like to express his gratitude to all technical staff at the Southampton Nanofabrication Centre (SNC) for their valuable assistance.

\section{REFERENCES}

[1] G. Masini, L. Colace, and G. Assanto, Si based optoelectronics for communications, Materials Science and Engineering B, vol. 89, no. 13, pp. 29, Feb. 2002.

[2] J. S. Orcutt, S. D. Tang, S. Kramer, K. Mehta, H. Li, V. Stojanovi, and R. J. Ram, Low-loss polysilicon waveguides fabricated in an emulated high-volume electronics process., Optics express, vol. 20, no. 7, pp. 724354, Mar. 2012.

[3] W. B. Jackson, Density of gap states of silicon grain boundaries determined by optical absorption, Applied Physics Letters, vol. 43, no. 2, p. 195,1983 .
[4] Q. Fang, J. F. Song, S. H. Tao, M. B. Yu, G. Q. Lo, and D. L. Kwong, Low Loss $(6.45 \mathrm{~dB} / \mathrm{cm})$ Sub-Micron Polycrystalline Silicon Waveguide Integrated with Efficient SiON Waveguide Coupler, Optics Express, vol. 16, no. 9, pp. 64256432, 2008.

[5] R. Takei, S. Manako, E. Omoda, M. Suzuki, M. Mori, Y. Sakakibara, and T. Kamei, Transmission Characteristics of Hydrogenated Microcrystalline Silicon Wire Waveguide at a Wavelength of $1.55 \mu \mathrm{m}$, Applied Physics Express, vol. 5, no. 8, p. 082501, Jul. 2012.

[6] K.-Y. Chan, D. Knipp, A. Gordijn, and H. Stiebig, Influence of crystalline volume fraction on the performance of high mobility microcrystalline silicon thin-film transistors, Journal of Non-Crystalline Solids, vol. 354, pp. 25052508, 2008.

[7] C. E. Richardson, Low-Temperature Hot-Wire Chemical Vapor Deposition of Epitaxial Films for Large- Grained Polycrystalline Photovoltaic Devices, California Institute of Technology, 2006. 